\title{
Influence of the Isotope Effect on the Charge-Exchange Process between Hydrogen Isotopes and Ions and Atoms of Plasma Facing Component Materials*)
}

\author{
Inga Yu. TOLSTIKHINA, Daiji KATO ${ }^{1)}$ and Vyacheslav P. SHEVELKO \\ P.N. Lebedev Physical Institute, Leninskii Prospekt 53, Moscow 119991, Russia \\ 1) National Institute for Fusion Science, 322-6 Oroshi-cho, Toki-city, Gifu 509-5292, Japan
}

(Received 8 December 2011 / Accepted 27 April 2012)

\begin{abstract}
The influence of the isotope effect (mass dependence) on the charge exchange process in low-energy collisions of ions and atoms sputtered from Plasma Facing Components (PFC) with hydrogen isotopes (H, D and T) is studied using the adiabatic theory of transitions in slow collisions developed by E. Solov'ev [Sov. Phys. Uspekhi 32, 228 (1989) [1]]. Results of the numerical calculations are presented for the charge-exchange cross sections of $\mathrm{Li}, \mathrm{Be}, \mathrm{C}$ ions colliding with hydrogen isotopes and for the inverse reactions.
\end{abstract}

(C) 2012 The Japan Society of Plasma Science and Nuclear Fusion Research

Keywords: slow collision, hydrogen isotope, isotope effect, charge-exchange cross section

DOI: $10.1585 /$ pfr.7.2401078

\section{Introduction}

The plasma-wall interactions remain one of the main areas of research in the physics and technology of controlled thermonuclear fusion. The conditions in the peripheral region largely determine also the processes in the main plasma. Numerical simulations of the near-wall and divertor region plasma in fusion devices are based on the knowledge of the rates of the elementary processes involving plasma particles and ions and atoms sputtered from PFC. The energies of collisions in the peripheral region are very low (about 1-500 eV/u). Under these conditions the charge-exchange becomes the dominant process for the neutralization and population of the excited states of plasma impurities and plays an important role in radiative cooling, particle transport and ions charge distribution. The isotope effect, found recently in charge-exchange reactions between hydrogen isotopes and alpha-particles [2-4] and PFC's ions ( $\mathrm{Li}, \mathrm{Be}, \mathrm{C}$ ) [5], manifests in a significant difference (up to several orders of magnitude) in the charge-exchange cross sections: the heavier the isotope the larger the cross section. This effect occurs at very low collision energies due to rotational interaction in close collisions - the scattering angle depends on reduced mass of colliding particles. Since DT burning plasma experiments are planned in ITER, hydrogen isotopic effects on transport of the sputtered particles is an important issue. We present here calculations of the charge-exchange cross sections in slow collisions of $\mathrm{Li}, \mathrm{Be}$ and $\mathrm{C}$ ions with $\mathrm{H}, \mathrm{D}$ and $\mathrm{T}$.

\footnotetext{
author'se-mail: inga@sci.lebedev.ru

*) This article is based on the presentation at the 21st International Toki Conference (ITC21).
}

\section{Theoretical Method}

We studied these reactions using the adiabatic approximation [1] which, in the theory of atomic collisions, is used to describe electronic transitions when the collision velocity is small and the nuclear motion can be treated classically. In this theory, there are no assumptions on the specific form of the electronic Hamiltonian, and only the smallness of the relative nuclear velocity is used. It results in a deeper understanding of the nature of non-adiabatic transitions. Since the isotope effect occurs at collision energies where the adiabatic theory applies, the adiabatic approximation is a natural theoretical framework for studying the effect. The cross sections are calculated with ARSENY code [6] based on the hidden crossing method.

Figure 1 shows the electronic potential curves for the charge exchange reaction between $\mathrm{Be}^{4+}$ ion and hydrogen calculated with ARSENY code. In the adiabatic theory transitions occur when the electronic wave function changes rapidly. It happens in the hidden crossings at the inter-nuclear distance where the electronic energies of two states are equal. There are two types of transitions - induced by radial and rotational couplings. Transitions caused by radial coupling are the Landau-Zener type of transitions and in Fig. 1 these 'radial' transitions are denoted by arrows (Rad) which connect initial $(\mathrm{H}(1 s)$ in separated atom limit) and final states of the electron and are positioned at real $R$ values of the corresponding hidden crossings. Radial coupling mixes the states with different principle and orbital quantum numbers and is responsible for the ionization. Rotational coupling associated with inter-nuclear axis rotation in close collisions induces transitions between the states which are degenerate in the united atom limit - they have the same principle and or- 


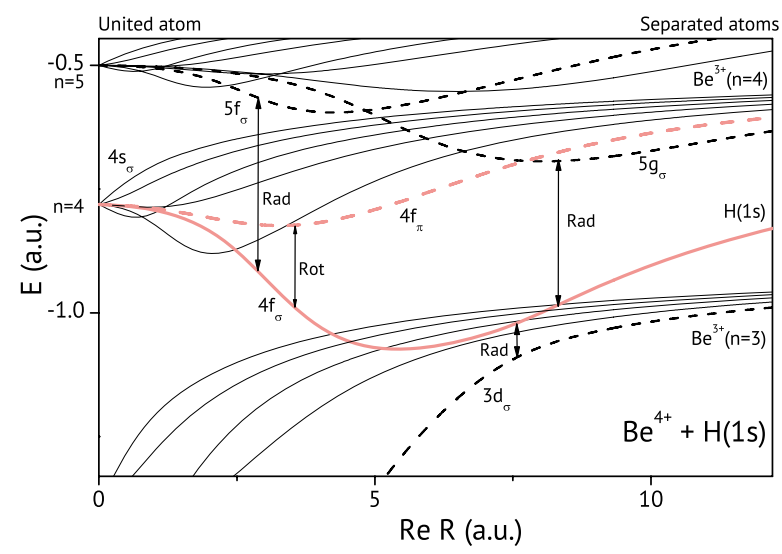

Fig. 1 (Color online) Potential curves describing the charge exchange process $\mathrm{Be}^{4+}+\mathrm{H}(1 s) \rightarrow \mathrm{Be}^{3+}(n l)+\mathrm{H}^{+}$as a function of the internuclear distance. Rotational transition $4 f_{\sigma}$ (red solid line) $-4 f_{\pi}$ (red dashed line) is the transition which causes the isotope effect in this reaction.

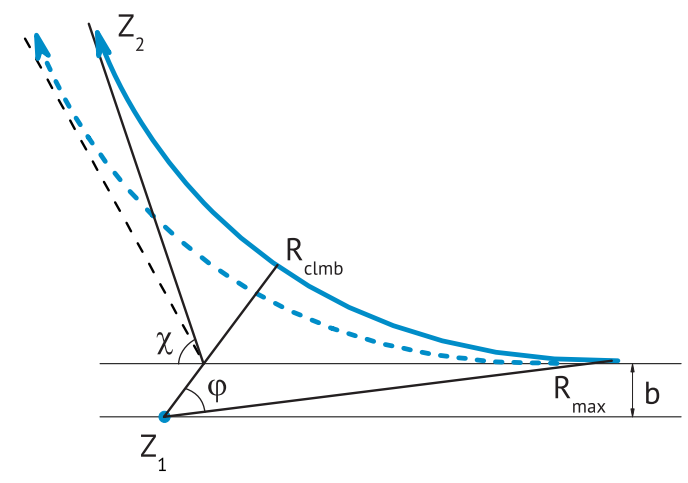

Fig. 2 (Color online) A Coulomb trajectory for close collisions.

bital quantum numbers and magnetic number $m$ changes by unity. Transitions caused by rotational coupling are the non Landau-Zener type of transitions since they occur in hidden crossing where the real part of inter-nuclear separation is zero. In Fig. 1 this transition is shown by arrows (Rot) positioned at arbitrary values of $R$. Rotational transition $4 f_{\sigma}-4 f_{\pi}$ is the transition which causes the isotope effect in the reaction $\mathrm{Be}^{4+}+\mathrm{H}(1 s), \mathrm{D}(1 s), \mathrm{T}(1 s)$.

To find the amplitude of the rotational transition the time-dependent Schrodinger equation is numerically solved with Coulomb trajectory in the united atom approximation. The boundary of the close collision region where the rotational interaction is taken into account (Fig. 2) is defined by the following equation:

$$
R_{\max }=\frac{\left(l+\frac{1}{2}\right)^{2}}{Z_{1}+Z_{2}}
$$

where $l$ is the orbital momentum of the electron, $Z_{1}$ and $Z_{2}$ are the charges of the nuclei. The trajectory can be expressed through the scattering angle $\chi$, angle of the inter-

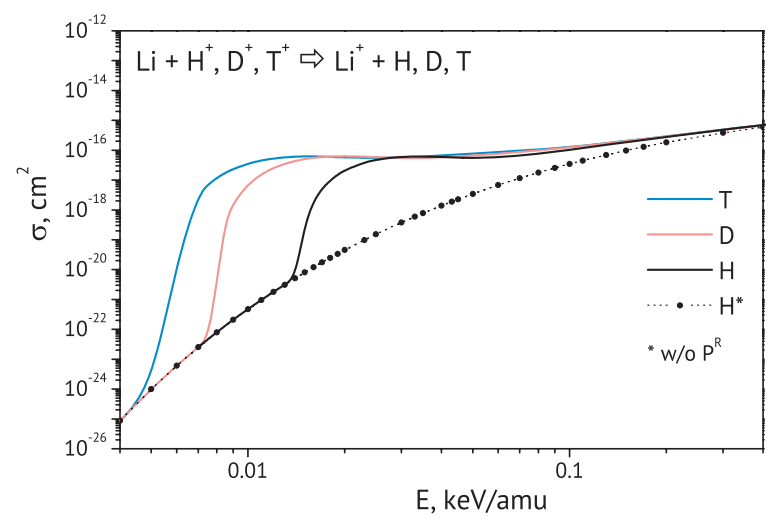

Fig. 3 (Color online) Total charge exchange cross sections as a function of collision energy calculated with and without taking into account the rotational interaction for the reaction $\mathrm{Li}\left(1 s^{2} 2 s\right)+\mathrm{H}^{+}\left(\mathrm{D}^{+}, \mathrm{T}^{+}\right)$.

nuclear axis rotation $\varphi$ and the impact parameter $\mathrm{b}$ :

$$
R=\frac{b \cos \frac{\chi}{2}}{\cos \varphi-\sin \frac{\chi}{2}}
$$

Since the scattering angle explicitly depends on the reduced mass $\mu$ of the colliding particles

$$
\chi=2 \arctan \left(\frac{Z_{1} Z_{2}}{\mu b v^{2}}\right),
$$

the trajectories for the different isotopes will be different - the heavier the isotope the smaller the internuclear separation corresponding to the closest approach. This in turn leads to a difference in the corresponding cross sections. The amplitude of the rotational transition is the solution of the time-dependent Schrodinger equation $\left(b<R_{\max }\right.$ and $R_{\text {max }}>R_{\text {clmb }}, R_{\text {clmb }}$ is the internuclear separation corresponding to the closest approach ):

$$
i \dot{a}_{m}-E_{m} a_{m}+i \sum_{m^{\prime}=-l}^{l}\left\langle\phi_{n l m}\left|\frac{\partial}{\partial t}\right| \phi_{n l m^{\prime}}\right\rangle a_{m^{\prime}}=0
$$

where

$$
\begin{aligned}
& E_{m}=3 \gamma m^{2} R^{2}, \\
& \gamma=\frac{Z_{1} Z_{2}\left(Z_{1}+Z_{2}\right)^{2}}{n^{3} l(l+1)(2 l-1)(2 l+1)(2 l+3)},
\end{aligned}
$$

and $\phi_{n l m}$ are the adiabatic wave functions. In the ARSENY code, they are expressed in terms of the spherical functions in the fixed coordinate system using the Wigner dfunctions [1].

\section{Results of Calculation and Discus- sion}

The total charge exchange cross section for the reactions $\mathrm{Li}+\mathrm{H}^{+}\left(\mathrm{D}^{+}, \mathrm{T}^{+}\right)$calculated with the ARSENY code is shown in Fig. 3. The cross section, designated as 'w/o $P^{R}$ ' in the figure, was calculated without taking into 


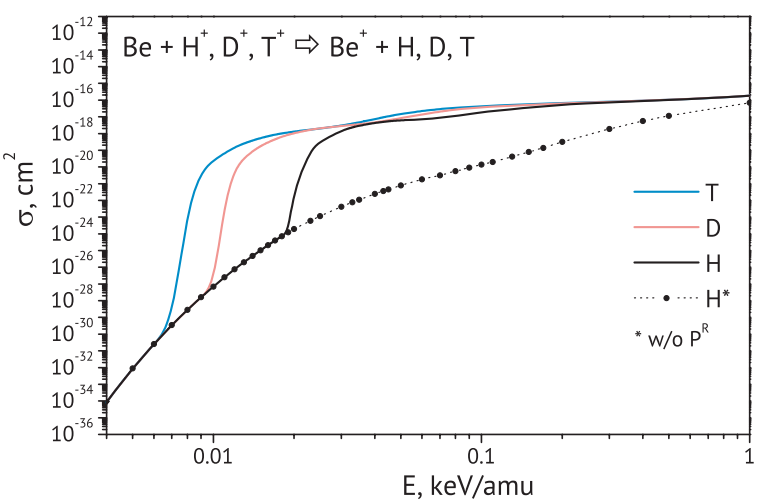

Fig. 4 (Color online) Total charge exchange cross sections as a function of collision energy calculated with and without taking into account the rotational interaction for the reaction $\mathrm{Be}\left(1 s^{2} 2 s^{2}\right)+\mathrm{H}^{+}\left(\mathrm{D}^{+}, \mathrm{T}^{+}\right)$.

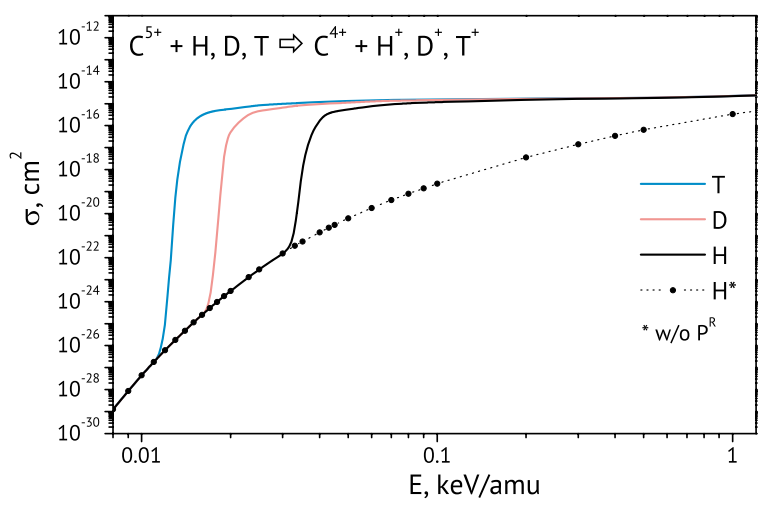

Fig. 5 (Color online) Total charge exchange cross sections as a function of collision energy calculated with and without taking into account the rotational interaction for the reaction $\left.\mathrm{C}^{5+}(1 s)+\mathrm{H}, \mathrm{D}, \mathrm{T}\right)$.

account the rotational interaction. As can be seen from the figure, the rotational coupling starts to contribute to the cross sections when the energy is decreased below $0.5 \mathrm{keV} / \mathrm{amu}$. This contribution is increasing with the energy decrease being larger for the heavier isotope. At the energies below $15 \mathrm{eV} / \mathrm{amu}$ the cross section, corresponding to the reaction with $\mathrm{H}^{+}$, is determined only by radial coupling while the main contribution to the $\mathrm{D}^{+}$and $\mathrm{T}^{+}$cross sections is still due to rotational interaction - colliding particles in the reactions with $\mathrm{D}^{+}$and $\mathrm{T}^{+}$are still in the region of close collisions where the rotational coupling occurs.

Figure 4 shows the total charge exchange cross sections for the reactions $\mathrm{Be}+\mathrm{H}^{+}\left(\mathrm{D}^{+}, \mathrm{T}^{+}\right)$. The rotational coupling starts to contribute to the cross sections when the energy is decreased below $1 \mathrm{keV} / \mathrm{amu}$ resulting in a significant isotope effect - the difference between the cross sections for the reactions with $\mathrm{H}^{+}$and $\mathrm{T}^{+}$for the collision energy $10 \mathrm{eV} / \mathrm{amu}$ is several orders of magnitude. The behavior of the charge exchange cross sections shown in Fig. 3 and Fig. 4 is non monotonous. It can be explained by the fact that two rotational transitions contribute to the total cross section. Two reactions discussed above are of interest for the modeling of the near-wall or diverter plasmas in fusion devices since $\mathrm{D}$ and $\mathrm{T}$ become neutral and can move to the core plasmas. The total charge exchange cross sections for the inverse reactions $\mathrm{C}^{5+}+\mathrm{H}(\mathrm{D}, \mathrm{T})$ is shown in Fig. 5. Only one rotational transition contributes to the total cross section resulting in its monotonous behavior.

These data were calculated with the effective charge for the initial or final states of the electron. For the calculation of the effective charge the CDW2 code [7] was used based on the distorted waves approximation.

\section{Summary and Conclusions}

The adiabatic theory was applied to study the mechanism of the isotope effect, its influence on the charge exchange cross sections and to calculate the collisional characteristics of the charge exchange processes involving hydrogen isotopes and their ions. The total cross sections were calculated for the charge exchange processes between $\mathrm{Li}, \mathrm{Be}$, and $\mathrm{C}$ ions and $\mathrm{H}, \mathrm{D}, \mathrm{T}$ atoms and for the inverse reactions using the ARSENY code based on the adiabatic approximation. The rotational transitions, which cause the isotope effect in the considered reactions, were defined and it was shown that if more than one channel with rotational transitions contribute to the final state then the crosssection behavior is not monotonous. Analyzing the data obtained one can conclude that the isotope effect becomes pronounced if the charge exchange reaction has resonant or quasi resonant channel, i.e. if the energies of the initial and the final states of the electron are exactly equal or differ by a very small value. In this case, the rotational transitions give the main contribution to the cross sections and the isotope effect can be observed.

The significant difference in the cross sections for the reactions with $\mathrm{H}, \mathrm{D}$ and $\mathrm{T}$ (several orders of magnitude) indicates the necessity for accounting the isotope effect in edge plasma modeling.

[1] E.A. Solov'ev, Sov. Phys. Uspekhi 32, 228 (1989).

[2] N. Stolterfoht, R. Cabrera-Trujillo, Y. Ohrn, E. Deumens, R. Hoekstra and J.R. Sabin, Phys. Rev. Lett. 99, 103201 (2007).

[3] N. Stolterfoht, R. Cabrera-Trujillo, P.S. Krstić, R. Hoekstra, Y. Öhrn, E. Deumens and J.R. Sabin, Phys. Rev. A 81, 052704 (2010).

[4] R. Cabrera-Trujillo, J.R. Sabin, Y. Öhrn, E. Deumens and N. Stolterfoht, Phys. Rev. A 83, 012715 (2011).

[5] I. Yu. Tolstikhina, D. Kato and V.P. Shevelko, Phys. Rev. A 84, 012706 (2011).

[6] E.A. Solov'ev, Workshop on Hidden Crossings in IonCollisions and in Other Nonadiabatic Transitions (Cambridge: MA: Harvard Smithonian Centre for Astrophysics) (1991).

[7] Dz. Belkic, R. Gayet and A. Salin, Comput. Phys. Commun. 23, 153 (1981). 